Güereca-Arvizuo, J.; Ramos-Jiménez, A.; Moreno-Brito, V.; Cervantes-Borunda, M.; HernándezTorres, R.P. (2020) Response of Creatine Kinase to an Anaerobic Supramaximal Exercise in ACTN3 Genotypes. Revista Internacional de Medicina y Ciencias de la Actividad Física y el Deporte vol. 20 (79) pp. 381-393 Http://cdeporte.rediris.es/revista/revista79/artefecto1172.htm

DOI: http://doi.org/10.15366/rimcafd2020.79.001

\title{
ORIGINAL
}

\section{RESPUESTA DE CREATINA QUINASA A UN EJERCICIO ANAEROBIO SUPRAMÁXIMO EN GENOTIPOS DE ACTN3}

\section{RESPONSE OF CREATINE KINASE TO AN ANAEROBIC SUPRAMAXIMAL EXERCISE IN ACTN3 GENOTYPES}

\section{Güereca-Arvizuo, J.'; Ramos-Jiménez, A. ${ }^{2}$; Moreno-Brito, V.; Cervantes- Borunda, M. ${ }^{4}$ y Hernández-Torres, R.P. ${ }^{4}$}

${ }^{1}$ Docente e investigador. Departamento de Ciencias de la Salud, División Multidisciplinaria en Ciudad Universitaria. Universidad Autónoma de Ciudad Juárez (México) jaime.guereca@uacj.mx

2 Docente e investigador. Departamento de Ciencias de la Salud, Instituto de Ciencias Biomédicas. Universidad Autónoma de Ciudad Juárez (México) aramos@uacj.mx

${ }^{3}$ Docente e investigador. Facultad de Medicina y Ciencias Biomédicas. Universidad Autónoma de Chihuahua (México) vmoreno@uach.mx

${ }^{4}$ Docentes e investigadores. Facultad de Ciencias de la Cultura Física. Universidad Autónoma de Chihuahua (México) mcervant@uach.mx, rhernant@uach.mx

AGRADECIMIENTOS: Los autores agradecen al Consejo Nacional de Ciencia y Tecnología (CONACYT) por la beca doctoral de Jaime Güereca Arvizuo (371390/249892).

Código UNESCO/UNESCO Code: 2409 Genética / Genetics; 5899 Deportes / Sports

Clasificación del Consejo de Europa/Council of Europe Classification: 2. Bioquímica del deporte / Biochemistry of sport

Recibido 28 de agosto de 2018. Received August 28, 2018 Aceptado 2 de febrero de 2019. Accepted February 2, 2019

\section{RESUMEN}

El objetivo del presente estudio fue investigar las diferencias en la actividad de la enzima Creatina Quinasa $(C K)$ en pre y post ejercicio anaerobio supramáximo (EASM) en portadores de los genotipos del gen de la alfa-actinina-3 (ACTN3). Se reclutaron 39 hombres sanos físicamente activos (18-35 años) y se sometieron a un EASM de $30 \mathrm{~s}$ (Wingate). El gen ACTN3 se determinó a partir del ADN de glóbulos blancos en sangre periférica y se evaluó la actividad de la CK en muestras 
sanguíneas en condiciones basales, a las 24 y $48 \mathrm{~h}$ post EASM. Los portadores del genotipo XX vs RR presentaron 1,4 veces menor actividad de CK en condiciones basales $(p<0,05)$ y una mayor actividad de CK a las $24 \mathrm{~h}$ post ejercicio $(p<0,05)$. Una serie de EASM fue capaz de causar un incremento significativo de la actividad de $\mathrm{CK}$ a las $24 \mathrm{~h}$ en los portadores del genotipo XX.

PALABRAS CLAVE: Alfa-actinina-3, creatina quinasa, genética deportiva, polimorfismo genético.

\section{ABSTRACT}

The aim of the present study was to investigate the differences in the activity of the enzyme Creatine Kinase (CK) in pre and post anaerobic supramaximal exercise (ASME) on carriers of the genotypes of the alpha-actinin-3 gene (ACTN3). 39 healthy physically active men (18-35 years) were enrolled and underwent an ASME of $30 \mathrm{~s}$ (Wingate). The ACTN3 gene was determined from the DNA of white blood cells in peripheral blood and the CK activity was evaluated in blood samples in basal conditions, at 24 and $48 \mathrm{~h}$ after of ASME. The carriers of genotype XX vs RR had 1.4 times lower CK activity in basal conditions $(p<0.05)$ and higher CK activity at $24 \mathrm{~h}$ after exercise $(p<0.05)$. A series of ASME was capable of causing a significant increase in CK activity at $24 \mathrm{~h}$ in the $\mathrm{XX}$ genotype carriers.

KEYWORDS: Alpha-actinin-3, creatine kinase, gene polymorphism, sports genetics.

\section{INTRODUCCIÓN}

El estudio de la genética en fechas actuales ha permitido conocer que la expresión de ciertos genes beneficia las capacidades físicas. No obstante, las capacidades físicas no están determinadas por un solo gen, sino por la sinergia e interacción de varios genes y la respuesta de estos a las condiciones ambientales (Saunders et al., 2007). A la fecha, se han estimado aproximadamente más de 200 variaciones genéticas relacionadas al rendimiento deportivo (Bray et al., 2009) pero, sólo poco más de 20 polimorfismos están estrechamente relacionados con el deportista de alto rendimiento (Bray et al., 2009). El polimorfismo R577X del gen de la alfaactinina-3 (ACTN3), es uno de los polimorfismos más estudiados en el ámbito del desempeño deportivo y de las capacidades físicas.

El gen ACTN3 presenta dos alelos, el alelo $\mathrm{R}$ codifica para la proteína funcional alfaactinina-3 y del alelo $X$ se obtiene una proteína alfa-actinina-3 no funcional (MacArthur y North, 2004). La proteína alfa-actinina-3 se expresa sólo en músculo esquelético, específicamente en las fibras de contracción rápida (Beggs et al., 1992), y es una de las principales componentes estructurales de la línea $Z$ del sarcómero (Beggs et al., 1992). La combinación de los alelos $\mathrm{R}$ y $X$ da origen a los genotipos RR, RX y $\mathrm{XX}$. Estos genotipos, han sido asociados con el rendimiento 
deportivo habiendo indicios de que proporcionan ventaja deportiva, ya sea de tipo aeróbica o anaeróbica (MacArthur y North, 2004). Dicho de otra manera, los genotipos RR y RX se asocian con una mayor capacidad de desarrollar potencia (Orysiak et al., 2014), fuerza (Broos et al., 2015) y velocidad (Mikami et al., 2014). Mientras que el genotipo XX favorece las actividades de resistencia aeróbica (Yang et al., 2003).

Se ha reportado que la proteína $A C T N 3$ tiene funciones de señalización metabólica en el músculo esquelético, al menos en modelo murino (Lee, Houweling, North y Quinlan, 2016), y por ello favorecer la fuerza muscular (MacArthur et al., 2008). Sin embargo, también se conoce que la ausencia de esta proteína (genotipo XX) no perjudica la contracción muscular (Zanoteli et al., 2002), pero provoca cambios a nivel enzimático en músculo esquelético de ratón $\mathrm{KO}$, modificando el metabolismo de las fibras rápidas, volviéndolas más oxidativas (MacArthur et al., 2008). Se reporta además una disminución en las propiedades elásticas de las proteínas musculares a nivel de la línea $Z$ del sarcómero (Seto et al., 2011), lo que se reflejaría en una menor capacidad de generar contracciones fuertes y rápidas. Con lo anterior podemos deducir que los portadores del genotipo XX serían más susceptibles al daño muscular provocado por contracciones fuertes y rápidas (Clarkson et al., 2005; Vincent et al., 2010) lo cual se vería reflejado en un incremento en la actividad de la Creatina Quinasa (CK).

Por otro lado, recientemente, se han reportado resultados inconsistentes en la actividad enzimática de la CK post ejercicio entre genotipos de ACTN3 (Clarkson et al., 2005; Vincent et al., 2010; Venckunas et al., 2012). En el ambiente deportivo, la CK, es utilizada como marcador bioquímico de daño tisular (Landau et al., 2012; Wu et al., 2004), sus concentraciones en plasma se incrementan posterior al ejercicio aeróbico (Hoffman, Ingwerson, Rogers, Hew-Butler y Stuempfle, 2012) o anaeróbico (Rodas et al., 2002; Vincent et al., 2010), siendo suficiente una sola sesión de ejercicio anaerobio supramáximo (EASM) para verla aumentada (Hammouda et al., 2012). Por lo anterior, el objetivo del presente trabajo fue investigar las diferencias en la actividad de la enzima CK pre y post EASM (Wingate) entre genotipos de ACTN3 en participantes físicamente activos.

\section{MATERIALES Y MÉTODOS}

\section{Participantes}

En un estudio transversal-observacional se reclutaron a 42 hombres sanos físicamente activos (edad 18-35 años) que realizaban actividades deportivas tres 0 más días a la semana. Los criterios de inclusión fueron: hombres saludables y físicamente activos. Los de exclusión: atletas de alto rendimiento, deportistas y participantes que estuvieran bajo algún tratamiento médico farmacológico, o que presentaran alguna lesión muscular que les impidiera realizar ejercicio físico. Antes de los estudios, a cada uno de los participantes se les explicaron los procedimientos y riesgos propios de las evaluaciones del estudio, posteriormente firmaron la carta 
de consentimiento informado. Para asegurarse del buen estado de salud de los participantes se les aplicó un cuestionario de salud general y el cuestionario PARQ \& YOU (Thomas et al., 1992). El protocolo de investigación y sus procedimientos fueron aprobados por el comité de ética de la Universidad Autónoma de Ciudad Juárez (CBE.ICB/053.08-15), basados en las recomendaciones de la declaración de Helsinki.

Todas las mediciones y determinaciones se realizaron de 8 a $10 \mathrm{~h}$, pidiendo a los participantes presentarse al laboratorio en condiciones de ayuno de al menos $8 \mathrm{~h}, \mathrm{y}$ sin haber practicado ejercicio y/o actividad física intensa durante 3 días previo al estudio.

\section{Ejercicio anaerobio supramáximo}

Antes de la aplicación del EASM se pesó al sujeto en traje de baño con una báscula digital (SECA 876, Hamburgo, Alemania). Posteriormente se aplicó un calentamiento general de 10 min incluyendo 5 min en bicicleta, para luego aplicarle el test anaeróbico de Wingate como EASM (Monark Ergomedic 884e, Suecia), de acuerdo con los procedimientos ya publicados (Bar-Or, 1987). La carga aplicada al cicloergómetro durante el test fue el $7,5 \%$ del peso corporal del participante.

\section{Determinación de la actividad enzimática de la CK}

Para determinar la cinética de la CK en plasma se pidió al participante presentarse durante 3 días consecutivos, en los cuales, a través de una punción en la vena antecubital, se obtuvo una muestra sanguínea antes de la aplicación del EASM, a las 24 y 48 h post EASM. La concentración de CK se determinó a través de un analizador de química clínica (Cobas Integra 400 plus, Roche Instrument Center, USA).

\section{Genotipos}

A través de un kit comercial MasterPure (Epicentre Biotechnolgies, USA) se obtuvo ADN genómico de leucocitos. Se amplificó un segmento de 291 pares de bases (pb) del gen ACTN3 aplicando la técnica de reacción en cadena de la polimerasa (PCR), se utilizaron los siguientes cebadores: directo: 5'-CTGTTGCC TGTGGTAAGTGGG-3' y reverso: 5'-GGTCACAGTATGCAGGAGGG-3' (Eurofins MWG-Operon, Alemania). La reacción de la PCR se realizó mezclando: $19 \mu \mathrm{L}$ de agua estéril, 2,5 $\mu \mathrm{L}$ de buffer, $0,75 \mu \mathrm{L}$ de $\mathrm{MgCl}_{2}, 0,5 \mu \mathrm{L}$ de dNTP's, 0,5 $\mu \mathrm{L}$ del cebador directo/reverso, una muestra de ADN a $100 \mathrm{ng}$ y 0,25 $\mu \mathrm{L}$ de la enzima Taq polimerasa, todo lo anterior sometido a 35 ciclos bajo las siguientes condiciones de amplificado: desnaturalización inicial de $95^{\circ} \mathrm{C}$ durante $10 \mathrm{~min}$ y de $95^{\circ} \mathrm{C}$ durante 1 min para la segunda desnaturalización. Para la etapa de alineación fue de $58^{\circ} \mathrm{C}$ durante $30 \mathrm{~s}$ y la etapa de elongación de $72^{\circ} \mathrm{C}$ durante $1 \mathrm{~min}$, una etapa de finalización a $72^{\circ} \mathrm{C}$ durante $10 \mathrm{~min}$. Para la determinación de los genotipos de ACTN3, se combinó el amplificado de PCR y la enzima Ddel (Desulfovibrio 
desulfuricans) (New England BioLabs, Estados Unidos) y se incubó a $37^{\circ} \mathrm{C}$ en calor húmedo durante $4 \mathrm{~h}$ y 20 min de inactivación a $65^{\circ} \mathrm{C}$. Se visualizaron los productos en geles de poliacrilamida al $12 \%$. Para el genotipo RR se obtuvo las siguientes bandas: 205 y 86 pb, mientras que para el genotipo RX: 205, 108, 97 y 86 bp, por último, para el genotipo XX: 108, 97 y 86 pb (Figura 1)

\section{Mediciones antropométricas, somatotipo y composición corporal}

Todas estas mediciones se realizaron de acuerdo con el protocolo ya publicado (Güereca et al., 2017) y siguiendo la metodología estandarizada por la International Society for the Advancement of Kinanthropometry (ISAK). Para determinar los componentes del somatotipo (Heath y Carter, 1990) y composición corporal se utilizó el software LifeSize 2,0 (Sidney, Australia) (Olds y Norton, 2000).

\section{Análisis estadísticos}

Para conocer las diferencias en las frecuencias genotípicas se realizó una Chi cuadrada $\left(\chi^{2}\right)$ para el total de la población (42 participantes). Sin embargo, 3 participantes no completaron la totalidad de las mediciones y fueron eliminados del estudio. El tamaño de la muestra se calculó con el programa G*power 3,1,9,2 (Faul, Erdfelder, Lang y Buchner, 2007), usando un tamaño del efecto de 0,50, un valor de alfa de 0,05 y un poder de 0,80 , sugiriendo una muestra total de 9 participantes por grupo. Para comprobar la normalidad de los datos se aplicó el test de Shapiro-Wilk, y se buscaron valores atípicos utilizando el diagrama de cajas y bigotes. En la búsqueda de diferencias entre genotipos, características antropométricas y actividad de CK, se aplicó un análisis de varianza (ANOVA) multivariante y la prueba post hoc de Tukey. Para analizar las diferencias en la actividad de la CK entre genotipos, entre tiempo y la posible influencia de la edad y masa muscular, se aplicó un ANOVA de medidas repetidas, colocando al genotipo como factor inter-sujetos, la edad y la masa muscular como covariable, seleccionándose el método Bonferroni para comparar los efectos principales. Por último, para eliminar la posible influencia de los valores basales en CK se realizó un ANOVA univariante de los residuales (24 y $48 \mathrm{~h}$ menos las concentraciones basales). El nivel de significancia estadística fue del 0,05. Los datos se analizaron con el programa SPSS versión 22,0.

\section{RESULTADOS}

Las frecuencias alélicas y genotípicas se presentan en la Tabla 1, observándose que la muestra poblacional se encuentra en equilibrio Hardy-Weinberg $\left(X^{2}=0,095\right.$; $p=0,75)$. 
Rev.int.med.cienc.act.fís.deporte - vol. 20 - número 79 - ISSN: 1577-0354

Tabla 1. Frecuencias genotípicas y alélicas del gen ACTN3.

\begin{tabular}{c|ccc|cc}
\hline \multirow{2}{*}{ Muestra } & \multicolumn{2}{|c|}{ Frecuencia genotípica n (\%) } & \multicolumn{2}{c}{ Frecuencia alélica \% } \\
\cline { 2 - 6 } & $\mathrm{RR}$ & $\mathrm{RX}$ & $\mathrm{XX}$ & $\mathrm{R}$ & $\mathrm{X}$ \\
\hline Hombres (n: 42) & $10(25)$ & $22(50)$ & $10(25)$ & 0,50 & 0,50 \\
\hline
\end{tabular}

$X^{2}=0,095 ; p=0,75$.

Las características físicas de los participantes entre genotipos fueron semejantes, excepto en la estatura (tabla 2), donde los participantes con genotipo RR fueron más altos que sus contrapartes. Los resultados de la actividad de CK se presentan al final de la Tabla 2.

Tabla 2. Características antropométricas y físicas de los sujetos agrupados por genotipos de ACTN3.

\begin{tabular}{lccc}
\hline & $\mathrm{RR}(\mathrm{n}=9)$ & $\mathrm{RX}(\mathrm{n}=20)$ & $\mathrm{XX}(\mathrm{n}=10)$ \\
\hline Edad (años) & $25,4 \pm 2,0$ & $23,8 \pm 2,7$ & $24,9 \pm 5,5$ \\
Peso $(\mathrm{kg})$ & $80,5 \pm 10,6$ & $77,7 \pm 13,4$ & $74,1 \pm 19,0$ \\
Talla $(\mathrm{m})$ & $1,79 \pm 0,05^{\mathrm{ab}}$ & $1,73 \pm 0,04$ & $1,72 \pm 0,08$ \\
IMC $\left(\mathrm{kg} / \mathrm{m}^{2}\right)$ & $24,8 \pm 2,8$ & $25,7 \pm 3,7$ & $24,5 \pm 5,0$ \\
Masa grasa $(\mathrm{kg})$ & $11,9 \pm 4,8$ & $12,5 \pm 5,1$ & $12,7 \pm 8,7$ \\
Grasa corporal $(\%)$ & $15,4 \pm 5,0$ & $16,3 \pm 4,8$ & $15,5 \pm 6,5$ \\
Masa muscular $(\mathrm{kg})$ & $31,7 \pm 4,6$ & $32,0 \pm 4,2$ & $31,4 \pm 7,1$ \\
Muslo medio $(\mathrm{cm})$ & $53,1 \pm 2,4$ & $53,4 \pm 4,6$ & $50,8 \pm 6,0$ \\
Endomorfia & $3,9 \pm 1,5$ & $4,9 \pm 1,8$ & $4,7 \pm 2,9$ \\
Mesomorfia & $4,2 \pm 0,9$ & $5,1 \pm 0,8$ & $4,9 \pm 1,3$ \\
Ectomorfia & $2,1 \pm 0,9$ & $1,5 \pm 1,0$ & $2,1 \pm 1,7$ \\
CK basal (UI/L) & $188,1 \pm 62,7 \mathrm{~b}$ & $149,9 \pm 48,8$ & $129,9 \pm 38,8$ \\
CK 24 h (UI/L) & $188,9 \pm 76,1$ & $172,6 \pm 63,4$ & $200,5 \pm 96,7$ \\
CK 48 h (Ul/L) & $200,6 \pm 99,6$ & $152,1 \pm 52,3$ & $145,4 \pm 47,0$ \\
\hline
\end{tabular}

Los valores se presentan en medias \pm desviación estándar (DE). IMC = Indice de masa corporal,

$\mathrm{CK}=$ Creatina Quinasa. ${ }^{a}$ diferencias entre genotipos RR vs RX. ${ }^{\circ}$ diferencias entre genotipos RR vs $X X . p<0,05$.

Los resultados de los fragmentos obtenidos de la restricción enzimática con la enzima Ddel para la determinación de los genotipos de ACTN3, se muestran en la Figura 1. 


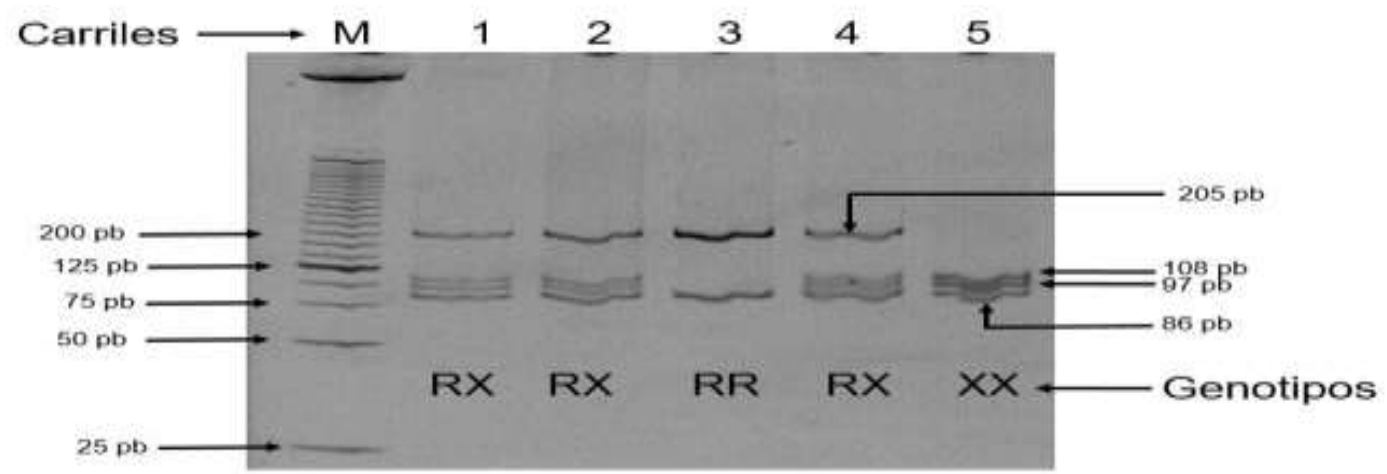

Figura 1. Fragmentos obtenidos de la restricción enzimatica con la enzima Ddel. Carril M: Marcadores de $25 \mathrm{pb}(1 \mathrm{\mu g} / \mu \mathrm{L})$. Se cargaron 2,5 HL a $150 \mathrm{ng} / \mu \mathrm{L}$. Carriles 1 al $5: 20 \mathrm{\mu L}$ de producto digerido de muestras representativas con buffer de carga $10 \times$ (azul de bromofenol). Flechas: Fragmentos de diferentes

tamaños obtenidos de la digestion de cada producto de amplificación. Gel de poliacrilamida/bisacrilamida al $12 \%$ tenido con bromuro de etidio (concentración final de $10 \mathrm{\mu g} / \mathrm{uL}$ ) en camara de electroforesis vertical grande $(25 \times 25 \mathrm{~cm})$ Hoefer $\mathrm{SB} 600$ Ruby (GE). Corrida electroforética: $145 \mathrm{~min}$ a $300 \mathrm{~V}$. M: Marcador molecular. pb: pares de base

Al analizar de manera individual las concentraciones iniciales (tiempo 0) de CK entre genotipos, se observó una menor concentración en el genotipo XX vs RR (Figuras 2 y 3). Al analizar de manera simultánea por medidas repetidas las diferencias en CK entre genotipo y tiempo, sólo se observaron diferencias entre tiempo, siendo mayor las concentraciones de CK a las $24 \mathrm{~h}$ respecto a los valores basales ( $p=$ 0,012); sin embargo, a través del análisis univariante de los residuales se observó que sólo en el genotipo XX vs RR la CK se elevó y presentó diferencias estadísticas a las $24 \mathrm{~h}$ post EASM (Figura 3).

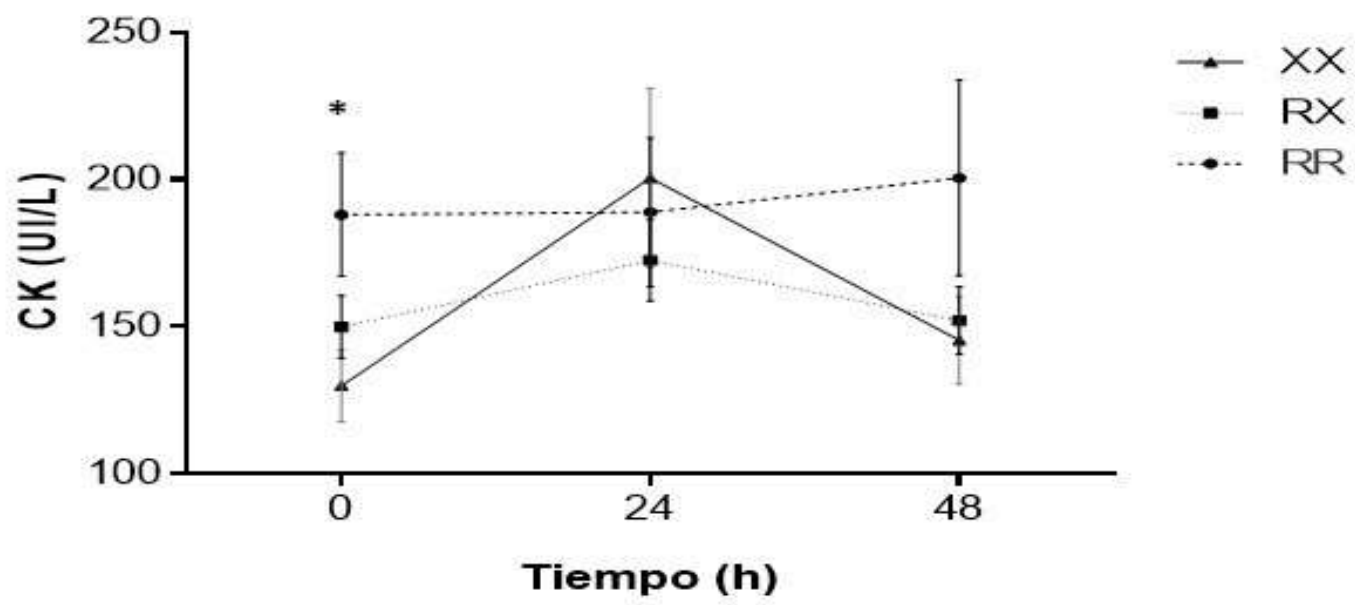

Figura 2. Comparación de la actividad de CK entre genotipos de ACTN3 en diferentes tiempos evaluados después de realizar una prueba de Wingate con el $7.5 \%$ del peso corporal agregado al pedaleo. Los valores se presentan en medias y error estándar. ${ }^{\star}$ Diferencia estadística entre XX y RR. CK: Creatina Quinasa. 


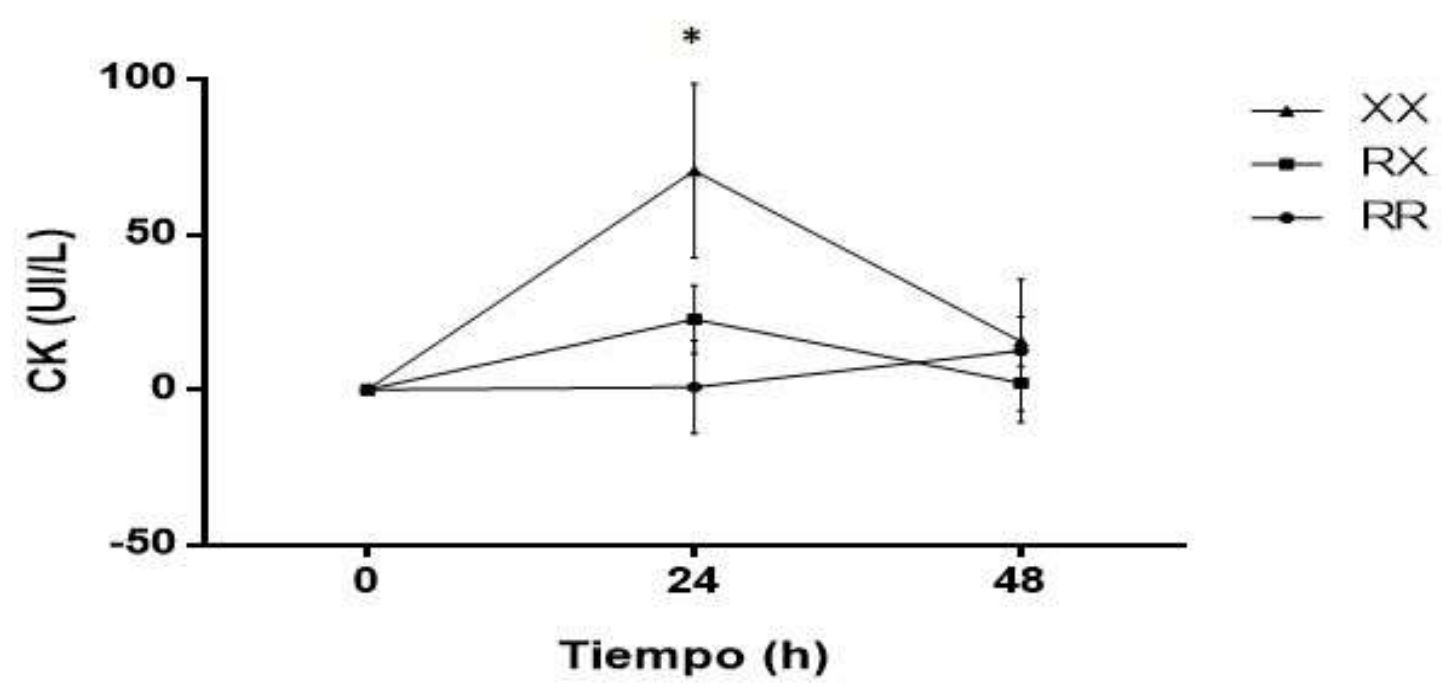

Figura 3. Comparación de la actividad de CK entre genotipos de ACTN3 en diferentes tiempos evaluados después de realizar una prueba de Wingate con el $7.5 \%$ del peso corporal agregado al pedaleo. Los valores se presentan en medias de los valores residuales y su error estándar. * Diferencia estadística entre XX y RR. CK: Creatina Quinasa.

\section{DISCUSIÓN}

Al eliminar la posible influencia de las condiciones basales de la actividad de CK por medio del análisis de los residuales, se observó que los portadores del genotipo XX vs RR presentan una mayor actividad a las $24 \mathrm{~h}$ post EASM, regresando a sus valores basales a las $48 \mathrm{~h}(p<0,05)$. Dichos resultados son similares a los reportados previamente por Vincent et al. (2010), quienes reportaron una tendencia no significativa, a las $24 \mathrm{~h}$ post ejercicio excéntrico. Lo anterior nos sugiere que los portadores del genotipo XX vs RR podrían ser más susceptibles a sufrir daño muscular después de una serie de EASM. Los presentes resultados están acordes a lo hipotetizado, pues se esperaba que los portadores de los genotipos XX vs RR presentarían una mayor actividad de la enzima CK post ejercicio. Se ha descrito que la actividad de $\mathrm{CK}$ en personas no entrenadas es mayor post ejercicio (Vincent y Vincent, 1997), sin embargo, en este trabajo no encontramos diferencias en las características físicas de los sujetos que pudieran influir en la actividad de CK. Se ha encontrado también que, en ratones, los portadores del genotipo XX presentan una disminución en las propiedades elásticas de las proteínas musculares a nivel de la línea $Z$ del sarcómero (Seto et al., 2011), provocando una disminución en las interacciones con otras proteínas musculares (Seto et al., 2011), lo que los haría más susceptibles al daño muscular durante el ejercicio (Clarkson et al., 2005). Por otro lado, se presume que la presencia del alelo $\mathrm{R}$ juega un papel de protección contra el daño muscular ante el incremento del ejercicio (Vincent et al., 2010)

Dado a las bajas concentraciones de CK encontradas a las 24 y $48 \mathrm{~h}$ post EASM (< $500 \mathrm{UI} / \mathrm{L}$ ), se considera que el ejercicio aplicado no fue de intensidad suficiente para provocar daño muscular (Martínez-Abat et al., 2005). Los resultados más altos de 
CK post ejercicio fueron de $200,5 \mathrm{UI} / \mathrm{L}$ en el genotipo XX, datos cercanos a los valores basales encontrados por otros autores (166-177 UI/L; Schumann y Klauke, 2003). Por lo anterior, una posible limitación del estudio fue no detectar el mejor momento para determinar la máxima actividad de la CK; sin embargo, hay contradicciones con relación al tiempo de detección del pico máximo de CK post ejercicio. Por ejemplo, en el trabajo de Pimenta et al. (2012) reportan la mayor actividad de $\mathrm{CK}$ a las $4 \mathrm{~h}$ post ejercicio, por el contrario, otros estudios mencionan que la mayor actividad se encuentra entre las 24 y $48 \mathrm{~h}$ post ejercicio (Vincent et al., 2010; Venckunas et al., 2012).

Por otro lado, el presente estudio muestra que los portadores del genotipo XX vs RR presentan menor actividad de CK en condiciones basales (1,4 veces menos), datos similares a los reportados por Clarkson et al. (2005). La menor actividad de CK observada en los portadores del genotipo XX podría ser debido al tipo de fibra muscular. Se ha reportado que los portadores del genotipo $X X$ vs RR y $R X$ presentan mayor proporción de fibras tipo I (Ahmetov et al., 2011), mientras que los portadores del genotipo RR vs XX, presentan mayor proporción de fibras musculares IIx (Vincent et al., 2010) y una mayor área seccional transversal en las fibras Ila y IIx (Broos et al., 2016) siendo las fibras tipo II las que contienen una mayor concentración de CK (Jansson, y Sylvén, 1985) debido a su metabolismo energético.

Se ha observado que la actividad de la CK se modifica por la edad, el género, la raza y la masa muscular (Baird, Graham, Baker, y Bickerstaff, 2012; Meltzer, 1971). Para determinar la posible influencia de la edad y la masa muscular sobre la actividad de la CK, se incluyeron como covariables en el modelo estadístico, sin embargo, no se observó la participación de ninguna de ellas.

Los estudios donde se relaciona la actividad de la CK y los genotipos de ACTN3 son escasos (Clarkson et al., 2005; Vincent et al., 2010; Venckunas et al., 2012), una de las principales diferencias entre dichos estudios y el presente trabajo, son los protocolos de ejercicio empleados para la estimulación de la CK. En el presente estudio se aplicó una sola sesión de EASM, mientras que Vincent et al. (2010) emplearon 20 repeticiones de contracciones excéntricas máximas de rodilla, Clarkson et al. (2005) emplearon 50 repeticiones de contracciones excéntricas máximas en flexores de codo, y Venckunas et al. (2012) emplearon 2 series de 50 saltos verticales. Dichos protocolos se basan en movimientos poco frecuentes durante la práctica de algún deporte de alto rendimiento, no obstante, y a pesar de utilizar protocolos anaeróbicos de ejercicio diferentes, ninguno de ellos estimuló incrementos significativos en la actividad de CK entre los genotipos.

Debido a que la prueba de Wingate aquí aplicada detectó poco incremento, pero significativo de CK a las $24 \mathrm{~h}$ post ejercicio, y solo en los portadores del genotipo $X X$, se sugiere un muestreo de la actividad de CK con intervalos más cortos entre las 4 y 24 h post EASM, además un protocolo de ejercicio más intenso y de mayor tiempo, ya que se ha observado que cuando el ejercicio es de mayor duración, las 
concentraciones de CK en plasma son mucho mayores (Belli et al., 2017; Del Coso et al., 2017).

\section{CONCLUSIONES}

El presente estudio muestra que los portadores del genotipo XX vs RR presentan menor actividad de CK en condiciones basales y una mayor actividad a las $24 \mathrm{~h}$ post EASM. Sin embargo, la intensidad, el tiempo y tipo de ejercicio aplicado no fueron suficientes para estimular una elevación de la CK superior a las $500 \mathrm{UI} / \mathrm{L}$, nivel sugerido como indicador de daño muscular.

\section{REFERENCIAS BILIOGRÁFICAS}

Ahmetov, I.I., Druzhevskaya, A.M., Lyubaeva, E.V., Popov, D.V., Vinogradova, O. L., and Williams, A.G. (2011). The dependence of preferred competitive racing distance on muscle fibre type composition and ACTN3 genotype in speed skaters. Exp Physiol. 96(12): 1302-1310. https://doi.org/10.1113/expphysiol.2011.060293

Baird, M.F., Graham, S.M., Baker, J.S., y Bickerstaff, G.F. (2012). Creatine-kinaseand exercise-related muscle damage implications for muscle performance and recovery. J Nutr Metab, 2012;2012:960363. https://doi.org/10.1155/2012/960363

Bar-Or, O. (1987). The Wingate anaerobic test an update on methodology, reliability and validity. Sports Medicine, 4(6), 381-394. https://doi.org/10.2165/00007256-198704060-00001

Beggs, A. H., Byers, T.J., Knoll, J.H., Boyce, F.M., Bruns, G.A., y Kunkel, L.M. (1992). Cloning and Characterization of Two Human Skeletal Muscle AlphaActinin Genes Located on Chromosomes 1 and 11. J Biol Chem, 267(13), 9281-9288.

Belli, T., Crisp, A. H., y Verlengia, R. (2017). Greater muscle damage in athletes with ACTN3 R577X (RS1815739) gene polymorphism after an ultra-endurance race: a pilot study. Biol Sport, 34(2), 105-110. https://doi.org/10.5114/biolsport.2017.64583

Bray, M.S., Hagberg, J.M., Pérusse, L., Rankinen, T., Roth, S.M., Wolfarth, B., y Bouchard, C. (2009). The human gene map for performance and healthrelated fitness phenotypes: the 2006-2007 update. Med Sci Sports Exerc, 41(1), 35-73. https://doi.org/10.1249/MSS.0b013e3181844179

Broos, S., Malisoux, L., Theisen, D., van Thienen, R., Ramaekers, M., Jamart, C., ..., and Francaux, M. (2016). Evidence for ACTN3 as a speed gene in isolated human muscle fibers. PLoS One. 11(3): 1-11. https://doi.org/10.1371/journal.pone.0150594

Broos, S., Van Leemputte, M., Deldicque, L., y Thomis, M. A. (2015). Historydependent force, angular velocity and muscular endurance in ACTN3 genotypes. Eur J Appl Physiol, 115, 1637-1643. https://doi.org/10.1007/s00421-015-3144-6 
Clarkson, P. M., Hoffman, E. P., Zambraski, E., Gordish-Dressman, H., Kearns, A., Hubal, M., ... y Devaney, J. M. (2005). ACTN3 and MLCK genotype associations with exertional muscle damage. J Appl Physiol, (1985), 99(2), 564-569. https://doi.org/10.1152/japplphysiol.00130.2005

Del Coso, J., Valero, M., Salinero, J.J., Lara, B., Díaz, G., Gallo-Salazar, C., ... y Cacabelos. (2017). ACTN3 genotype influences exercise-induced muscle damage during a marathon competition. Eur J Appl Physiol, 117(3), 409-416. https://doi.org/10.1007/s00421-017-3542-z

Faul, F., Erdfelder, E., Lang, A.G., y Buchner, A. (2007). G*Power 3: a flexible statistical power analysis program for the social, behavioral, and biomedical sciences. Behav Res Methods, 39(2), 175-191. https://doi.org/10.3758/BF03193146

Güereca-Arvizuo, J., Ramos-Jiménez, A., Flores-Martínez, N., Reyes-Leal, G y Hérnandez-Torres, R.P. (2017). ACTN3 genotypes and their association with athletes somatotype: Results of a pilot study. ECORFAN-Ecuador Journal 2017 4(6), 10-17.

Hammouda, O., Chtourou, H., Chaouachi, A., Chahed, H., Ferchichi, S., Kallel, C., ... y Souissi, N. (2012). Effect of short-term maximal exercise on biochemical markers of muscle damage, total antioxidant status, and homocysteine levels in football players. Asian $J$ Sports Med, 3(4), 239-246. https://doi.org/10.5812/asjsm.34544

Heath, B., and Carter, J.E. (1990). Somatotyping Development and Applications. Cambridge university press.

Hoffman, M. D., Ingwerson, J. L., Rogers, I. R., Hew-Butler, T., y Stuempfle, K. (2012). Increasing creatine kinase concentrations at the $161-\mathrm{km}$ Western States Endurance Run. Wildernees Environ Med, 23(1), 56-60. https://doi.org/10.1016/i.wem.2011.11.001

Jansson, E., y Sylvén, C. (1985). Creatine kinase MB and citrate synthase in type I and type II muscle fibres in trained and untrained men. Eur $J$ Appl Physiol Occup Physiol, 54(2), 207-209. https://doi.org/10.1007/BF02335931

Landau, M. E., Kenney, K., Deuster, P., Gonzalez, R. S., Contreras-Sesvold, C., Sambuughin, N., O'Connor, F. G., y Cambell, W. (2012). Investigation of the relationship between serum creatine kinase and genetic polymorphisms in military recruits. Military Medicine, 177(11), 1359-1365. https://doi.org/10.7205/MILMED-D-12-00086

Lee, F., Houweling, P., North, K., y Quinlan, K. (2016). How does alfa-actinin-3 deficiency alter muscle function? Mechanistic insights into ACTN3, the gene for speed. (2016). Biochimica et Biophysica Acta, 1863. 868-893. https://doi.org/10.1016/i.bbamcr.2016.01.013

MacArthur, D. G., y North, K. N. (2004). A gene for speed? The evolution and function of a-actinin-3. Bioessays, 26(7), 786-795. https://doi.org/10.1002/bies.20061

MacArthur, D. G., Seto, J. T., Chan, S., Quinlan, K. G., Raftery, J. M., Turner, N., ... y North, K. N. (2008). An Actn3 knockout mouse provides mechanistic insights into the association between alpha-actinin-3 deficiency and human athletic 
Rev.int.med.cienc.act.fís.deporte - vol. 20 - número 79 - ISSN: 1577-0354

performance. Hum Mol Genet, 17(8), 1076-1086. https://doi.org/10.1093/hmg/ddm380

Martínez-Abat, A., Boulaiz, H., Prados, J., Marchal, J., Padial, P., Caba, O., Rodriguez-Serrano, F., Aranega, A. (2005). Release of A-Actin into serum after skeletal muscle damage. Br $J$ Sports Med, 39(11), 830-834). https://doi.org/10.1136/bjsm.2004.017566

Meltzer, H.Y. (1971). Factors affecting serum creatine phosphokinase levels in the general population: the role of race, activity and age. Clin Chim Acta, 33, 165172. https://doi.org/10.1016/0009-8981(71)90264-6

Mikami, E., Fuku, N., Murakami, H., Tsuchie, H., Takahashi, H., Ohiwa, N., ... y Tanaka, M. (2014). ACTN3 R577X Genotype is Associated with Sprinting in Elite Japanese Athletes. Int $J$ Sports Med, 35(2), 172-177. https://doi.org/10.1055/s-0033-1347171

Olds, T., Norton, K., and Clark, J. (2000). LifeSize user manual release 1.0 Educational Software for Body Composition Analysis. Australia: Human Kinetics, Inc.

Orysiak, J., Busko, K., Michalski, R., Mazur-Różycka, J., Gajewski, J., MalczewskaLenczowska, J., ... y Pokrywka, A. (2014). Relationship between ACTN3 R577X polymorphism and maximal power output in elite Polish athletes. Medicina, 50(5), 303-308. https://doi.org/10.1016/i.medici.2014.10.002

Pimenta, E. M., Coelho, D. B., Cruz, I. R., Morandi, R. F., Veneroso, C. E., de Azambuja Pussieldi, G., Carvalho, M. R., Silami-Garcia, E., De Paz Fernández, J. A. (2012). The ACTN3 genotype in soccer players in response to acute eccentric training. Eur $J$ Appl Physiol, 112(4), 1495-1503. https://doi.org/10.1007/s00421-011-2109-7

Rodas, G., Ventura, J. L., Cadefau, J. A., Cussó, R., y Parra, J. (2002). Un programa de entrenamiento intenso para un rápido mejoramiento tanto del metabolismo aeróbico como del anaeróbico. Apunts Medicina de l'Esport, 37(140), 5-12. https://doi.org/10.1016/S1886-6581(02)76031-3

Saunders, C. J., September, A. V., Xenophontos, S. L., Cariolou, M. A., Anastassiades, L. C., Noakes, T. D., y Collins, M. (2007). No association of the ACTN3 gene R577X polymorphism with endurance performance in Ironman Triathlons. Ann Hum Genet, 71(Pt 6), 777-781. https://doi.org/10.1111/j.1469-1809.2006.00385.x

Schumann, G., y Klauke, R. (2003). New IFCC reference procedures for the determination of catalytic activity concentrations of five enzymes in serum: preliminary upper reference limits obtained in hospitalized subjects. Clin Chim Acta, 327(1-2), 69-79. https://doi.org/10.1016/S0009-8981(02)00341-8

Seto, J., Lek, M., Quinlan, K., Houweling, J., Zheng, X., Garton, F., ... North, N. (2011). Deficiency of alfa-actinin-3 is associated with increased susceptibility to contraction-induced damage and skeletal muscle remodeling. Human molecular genetics, 20(15), 2914-2927. https://doi.org/10.1093/hmg/ddr196

Thomas, S., Reading, J., y Shephard, R.J. (1992). Revision of the Physical Activity Readiness Questionnaire (PAR-Q). Can J Spt Sci, 17(4), 338-345.

Venckunas, T., Skurvydas, A., Brazaitis, M., Kamandulis, S., Snieckus, A., y Moran, C. N. (2012). Human alpha-actinin-3 genotype association with exercise- 
induced muscle damage and the repeated-bout effect. Appl. Physiol.Nutr. Metab, 37, 1-9. https://doi.org/10.1139/h2012-087

Vincent, H.K., Vincent, K.R. (1997). The effect of training status on the serum creatine kinase response, soreness and muscle function following resistance exercise. Int J Sports Med 1997, 18(6), 431-437. https://doi.org/10.1055/s2007-972660

Vincent, B., Windelinckx, A., Nielens, H., Ramaekers, M., Van Leemputte, M., Hespel, P., y Thomis, M. A. (2010). Protective role of $\alpha$-actinin-3 in the response to an acute eccentric exercise bout. J Appl Physiol (1985), 109(2), 564-573. https://doi.org/10.1152/japplphysiol.01007.2009

Wu, H. J., Chen, K. T., Shee, B. W., Chang, H. C., Huang, Y. J., y Yang, R. S. (2004). Effects of $24 \mathrm{~h}$ ultra-marathon on biochemical and hematological parameters. World J Gastroenterol, 10(18), 2711-2714. https://doi.org/10.3748/wjg.v10.i18.2711

Yang, N., MacArthur, D.G., Gulbin, J., Hahn, A., Beggs, A.H., Eastel, S., y North, K. (2003). ACTN3 Genotype Is Associated with Human Elite Athletic Performance. Am J Hum Genet, 73(3), 627-631. https://doi.org/10.1086/377590

Zanoteli, E., Lotuffo, R., Oliveira, A., Beggs, A. H., Canovas, M., Zatz, M., y Vainzof, M. (2002). Deficiency of Muscle a-Actinin-3 is compatible with high muscle performance. $J \mathrm{Mol}$ https://doi.org/10.1385/JMN:20:1:39

Neurosci, 20(1),

$39-42$

Número de citas totales / Total references:37 (100\%)

Número de citas propias de la revista /Journal's own references:0 (0\%)

Rev.int.med.cienc.act.fís.deporte- vol. X - número X - ISSN: 1577-0354 